\title{
Hepatic Scalloping - A Rare Presentation of Abdominal Tuberculosis
}

\author{
Sarika Goel ${ }^{1}$, Ankur Malhotra², Shruti Chandak ${ }^{3}$, Atul Pratap Singh ${ }^{4}$, Ashwani Jain ${ }^{5}$, Deepti Arora ${ }^{6}$ \\ ${ }^{1}$ Resident, Department of Radiodiagnosis, Teerthanker Mahaveer Medical College \& Research Centre (TMMC\&RC), \\ Moradabad, Uttar Pradesh, ${ }^{2}$ Associate Professor, Department of Radiodiagnosis, Teerthanker Mahaveer Medical College \& \\ Research Centre (TMMC\&RC), Moradabad, Uttar Pradesh, ${ }^{3}$ Professor, Department of Radiodiagnosis, Teerthanker Mahaveer \\ Medical College \& Research Centre (TMMC\&RC), Moradabad, Uttar Pradesh, ${ }^{4}$ Resident, Department of Radiodiagnosis, \\ Teerthanker Mahaveer Medical College \& Research Centre (TMMC\&RC), Moradabad, Uttar Pradesh, ${ }^{5}$ Resident, Department \\ of Radiodiagnosis, Teerthanker Mahaveer Medical College \& Research Centre (TMMC\&RC), Moradabad, Uttar Pradesh, \\ ${ }^{6}$ Associate Professor, Department of Pathology, TeerthankerMahaveer Medical College \& Research Centre (TMMC\&RC), \\ Moradabad, Uttar Pradesh- 244001, India.
}

Corresponding author: Dr. Ankur Malhotra, Associate Professor, Department of Radiodiagnosis, Teerthanker Mahaveer Medical College \& Research Centre (TMMC\&RC), Moradabad, Uttar Pradesh- 244001, India.

DOI: http://dx.doi.org/10.21276/ijcmsr.2020.5.2.10

How to cite this article: Sarika Goel, Ankur Malhotra, Shruti Chandak, Atul Pratap Singh, Ashwani Jain, Deepti Arora. Hepatic scalloping - a rare presentation of abdominal tuberculosis. International Journal of Contemporary Medicine Surgery and Radiology. 2020;5(2):B44-B46.

\section{A B S T R A C T}

Introduction: Peritoneal involvement is the most common feature of abdominal tuberculosis (TB).CT findings of peritoneal TB include peritoneal thickening, ascites with intestinal adhesions, necrotic lymph nodes, omental caking and rarely visceral scalloping. Scalloping of visceral organs is described in pseudomyxoma peritonei, peritoneal carcinomatosis, and malignant ascites but not in tuberculosis.

Case report: Here we report one such unusual case where a male patient presented with fever and abdominal pain, went through a set of investigations such as montoux test, chest radiograph, pleural fluid analysis, USG and CT abdomen, to attain a diagnosis of abdominal tuberculosis and was found to have visceral scalloping on CT abdomen as a rare radiological finding.

Conclusion: Complete clinical picture with specific laboratory findings can help in achieving the accurate diagnosis in such cases.

Keywords: Hepatic Scalloping; Abdominal Tuberculosis; Pseudomyxoma Peritonei, Peritoneal Carcinomatosis

\section{INTRODUCTION}

Tuberculosis can present in varied forms viz. pulmonary tuberculosis or extra pulmonary tuberculosis. Lungs are the primary involved organs and abdominal involvement occurs in about $11-12 \%$ of patients with extra-pulmonary tuberculosis. ${ }^{1}$

Peritoneal and pleural effusions due to tuberculosis (TB) occur most commonly due to hematogenous spread from previous pulmonary $\mathrm{TB}$, but it may be secondary to lymph node rupture, gastrointestinal dissemination or tubal involvement. $^{2}$

Peritoneal tuberculosis is the commonest form of abdominal tuberculosis and the diagnosis is suspected in setting of clinical features like abdominal pain and distension with radiological findings like presence of ascites, lymphadenopathy, mesenteric \& peritoneal thickening and visceral scalloping. ${ }^{3}$ Here we report a case of peritoneal tuberculosis with a rare finding of hepatic scalloping that is more commonly associated with pseudomyxoma peritonei and peritoneal carcinomatosis.

\section{CASE REPORT}

A 24 year male patient presented to emergency department with fever and abdominal pain for past three months, accompanied by decreased appetite and weight loss. The patient had a past history of seizure disorder and was on regular treatment. On physical examination the patient had tenderness in lower abdomen without any guarding or rigidity.

Laboratory findings were as follows: Haemoglobin 10.4 g/dL (normal, 13-17), Total leucocytes count 7.9x109 /L (normal, 4-11), Platelets $390 \times 10^{9} / \mathrm{L}$ (normal, 150-400), ESR $11 \mathrm{~mm} / \mathrm{hr}$ and negative viral markers (HCV, HBsAg \& HIV test). Mantoux test was positive with induration of $25 \mathrm{~mm}$ and high pleural fluid adenosine deaminase (ADA) of 32.4 IU/L (normal 0-30).

Chest radiograph showed blunting of the right costophrenic angle due to a small pleural effusion without any parenchymal abnormality.

Ultrasound whole abdomen demonstrated mild hepatosplenomegaly, mild pleural effusion and enlarged 


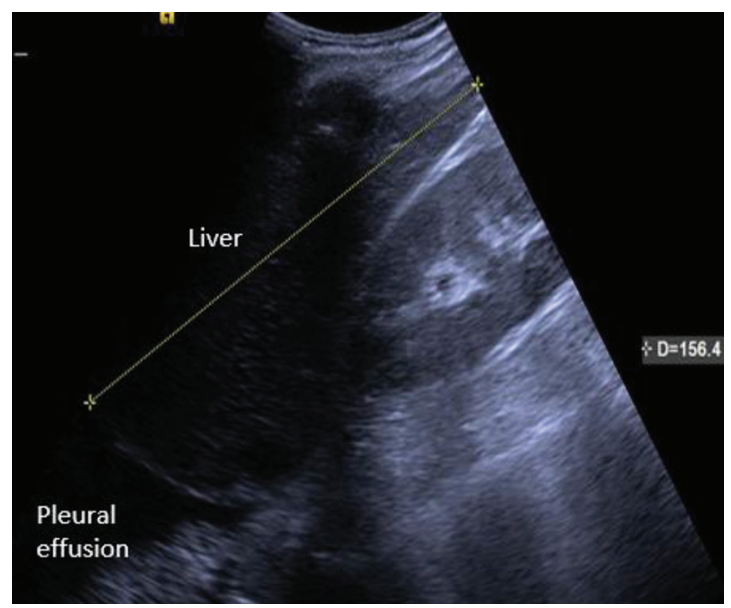

Figure-1: Gray scale abdominal ultrasound shows mildly enlarged liver and right sided mild pleural effusion.

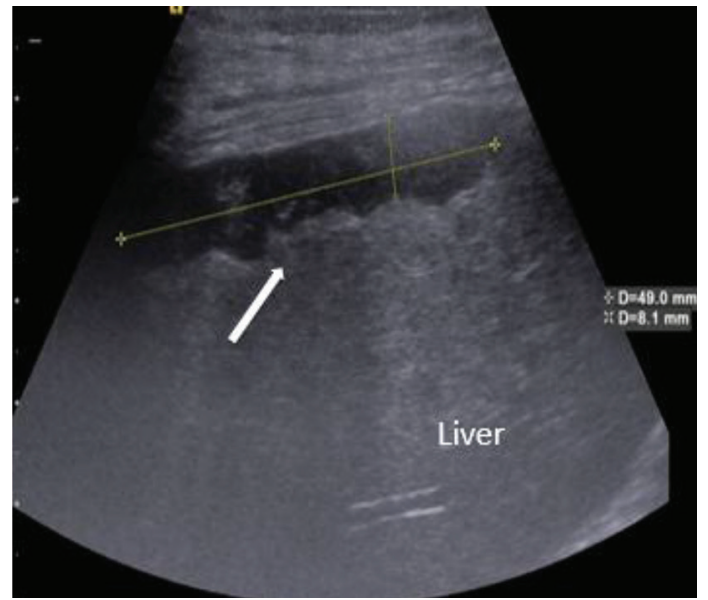

Figure-2: Gray scale abdominal ultrasound shows hypoechoic areas in the subcapsular region of right lobe of liver causing indentation over underlying hepatic margins (white arrow).

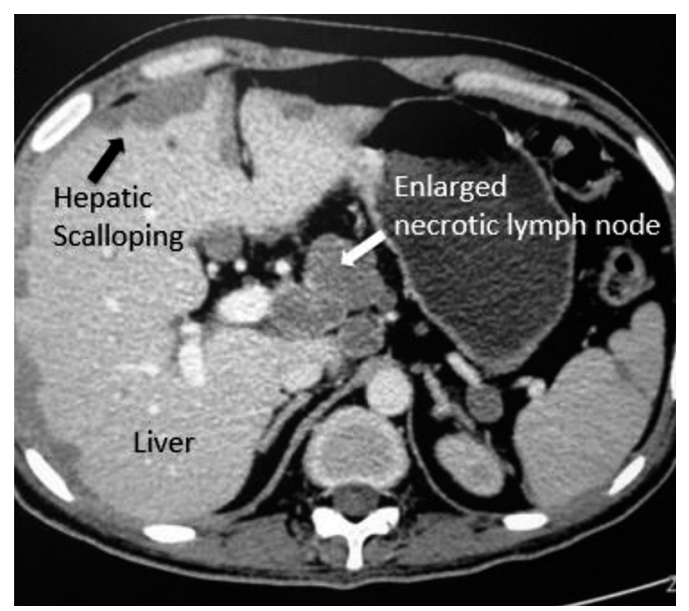

Figure-3: Axial Contrast Enhanced CT image shows non enhancing subcapsular hypodensities causing hepatic scalloping with multiple discrete enlarged necrotic lymph nodes at porta.

abdominal lymph nodes with no ascites (Fig 1). Note was also made of few hypoechoic cystic areas in sub capsular region of right lobe of liver indenting the underlying liver

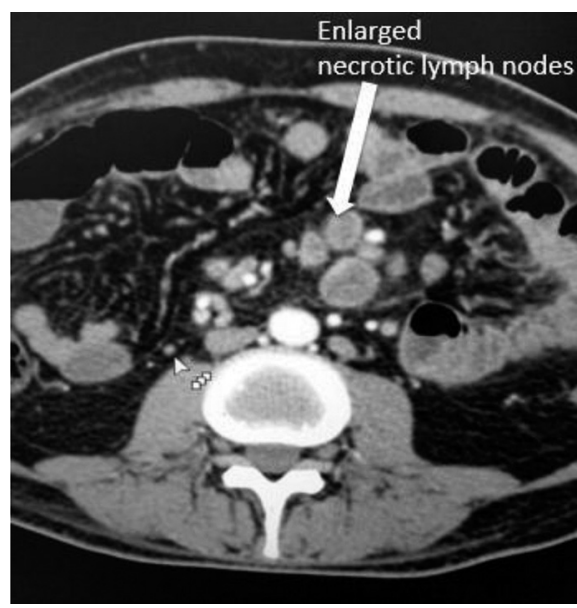

Figure-4: Axial Contrast Enhanced CT image shows multiple discrete enlarged necrotic mesenteric lymph nodes

surface (Fig 2). Abdominal contrast enhanced computed tomography (CECT) showed liver scalloping with multiple enlarged necrotic abdominal nodes (Fig $3 \& 4$ ). FNAC from the hepatic lesion showed only caseating necrosis with no evidence of any epithelioid cell granuloma. AFB stain was positive for tubercular bacilli.

\section{DISCUSSION}

The diagnosis of extra-pulmonary TB can be difficult, due to lack of characteristic imaging signs and its insidious nonspecific presentation, leading to delays in treatment. CT findings of peritoneal TB include peritoneal \& mesenteric thickening, ascites with septations, omental caking and rarely hepatic scalloping. ${ }^{4}$

Hepatic scalloping mainly in sub capsular region is characterized by indentations of the visceral margins as a consequence of pressure by intraperitoneal collection and is typically described in cases of peritoneal carcinomatosis, pseudomyxoma peritonei and rarely in peritoneal tuberculosis, but can be caused by other benign \& malignant pathological conditions. $^{5}$

Pseudomyxoma peritonei is mucinous accumulation within the peritoneal cavity that is associated with a malignant neoplasm from gastrointestinal tract most commonly arising from the colon, appendix or ovary. Mucin or gelatinous material is mostly low in CT attenuation, but areas of softtissue attenuation may be present that represent solid tumour elements, fibrosis, or compression of the mesentery. ${ }^{3}$

Scalloping of the visceral surfaces of the intraperitoneal organs is an important diagnostic finding that helps differentiate pseudomyxoma from simple ascites (exudative/ transduative). It is most commonly observed along the margins of the liver and spleen. Pseudomyxoma peritonei does not involve the visceral organs by haematogenous routes and scalloping of liver surface in cases of pseudomyxoma peritonei is due to pressure effect of mucinous/gelatinous material from the peritoneal deposits in the peri-hepatic region. This is in contrast to tubercular involvement of the solid organs resulting in intra-parenchymal granulomatous lesions. ${ }^{6}$

The imaging findings of peritoneal carcinomatosis vary from multifocal discrete nodules to infiltrative masses. Infiltration 
of the bowel mesentery with carcinomatosis may produce characteristic stellate or pleated patterns that occurs as the soft-tissue tumor replaces the normal mesenteric fat. ${ }^{3}$

Fibrous variety of peritoneal TB shows remarkable omental thickening, entanglement of bowel loops \& occasionally loculated ascites which mimics peritoneal carcinomatosis. However in case of peritoneal TB the hepatic scalloping is due to indentation of hepatic surface by sub capsular inflammatory granulomas. The radiological appearance in both these cases will remain similar in ultrasound as well as CT. ${ }^{7,8}$

Visceral scalloping should not always be related to malignant ascites/peritoneal carcinomatosis. It can be associated with abdominal tuberculosis, though rarely (as seen in this case). ${ }^{3,4}$

\section{CONCLUSION}

Hepatic scalloping, although described primarily in peritoneal carcinomatosis or pseudomyxoma peritonei may sometimes be seen in cases of peritoneal tuberculosis as well. Thus hepatic scalloping should not be used as sole discriminating criteria for differentiating these pathologies. In a rare case of 'abdominal tuberculosis', hepatic scalloping can be encountered.

\section{REFERENCES}

1. Kapoor VK. Abdominal tuberculosis. Postgrad Med J. 1998;74(2):459-467.

2. Burrill J, Williams CJ, Bain G, et al. Tuberculosis: a radiologic review. Radiographics. 2007;27(5):12551273.

3. Sharma V, Bhatia A, Malik S, Singh N, Rana SS. Visceral scalloping on abdominal computed tomography due to abdominal tuberculosis. TherAdv Infect Dis. 2017;4(1):3-9.

4. Sintra SN, Madaleno J, Canha C, Simão A, Carvalho A. Liver scalloping - An unusual presentation of a benign disease. Int $\mathrm{J}$ Case Rep Images. 2019;10(6):101001Z01SS2019.

5. Deshpande SS, Joshi AR, Deshpande SS, Phajlani AS. Computed tomographic features of abdominal tuberculosis: Unmask the impersonator! AbdomRadiol. 2019;44(1):11-21.

6. Levy AD, Shaw JC, Sobin LH. Secondary tumors and tumorlike lesions of the peritoneal cavity: Imaging features with pathologic correlation. Radiographics 2009;29(2):347-73.

7. Gulati MS, Sarma D and Paul SB. CT appearances in abdominal tuberculosis. A pictorial essay. Clin Imaging. 1999;23(5):51-59.

8. Na-Chiang Mai W, Pojchamarnwiputh S, Lertprasertsuke N, Chitapanarux T. CT findings of tuberculous peritonitis. Singapore Med J. 2008;49(3):488-491.

Source of Support: Nil; Conflict of Interest: None

Submitted: 24-03-2020; Accepted: 28-04-2020; Published online: 29-05-2020 